EUROPEAN ORGANIZATION FOR NUCLEAR RESEARCH

European Laboratory for Particle Physics

Large Hadron Collider Project

LHC Project Report 507

\title{
UPDATE OF A COOLDOWN AND WARMUP STUDY FOR THE LARGE HADRON COLLIDER
}

\author{
L. Liu ${ }^{1}$, G. Riddone ${ }^{2}$ and L. Tavian ${ }^{2}$
}

\begin{abstract}
The paper presents the inventory of components and materials for LHC magnets, especially for main dipoles and quadrupoles. A mathematical model for LHC transient modes, such as cooldown and warmup of a magnet, a standard cell and the eight LHC sectors, has been developed on the basis of the up-to-date layout of the LHC machine, and validated by experimental data. The model considers the momentum and continuity equations, as well as the energy equations for helium and materials. Based on the simulation results, the heat transfer in the magnets has been studied and the transient modes optimized.
\end{abstract}

1 Cryogenic Laboratory, Chinese Academy of Sciences, Beijing, China

2 CERN, LHC Division

Presented at the 2001 Cryogenic Engineering Conference and International Cryogenic Materials Conference CEC/ICMC 2001

16-20 July 2001, Madison, Wisconsin, USA

Administrative Secretariat

LHC Division

CERN

CH - 1211 Geneva 23

Switzerland

Geneva, 19 October 2001 


\title{
UPDATE OF A COOLDOWN AND WARMUP STUDY FOR THE LARGE HADRON COLLIDER
}

\author{
L. Liu ${ }^{1}$, G. Riddone ${ }^{2}$, and L. Tavian ${ }^{2}$ \\ ${ }^{1}$ Cryogenic Laboratory, Chinese Academy of Sciences, \\ P. O. Box 2711, Beijing 100080, China \\ ${ }^{2}$ LHC Division, CERN, \\ CH-1211 Geneva 23, Switzerland
}

\begin{abstract}
The paper presents the inventory of components and materials for LHC magnets, especially for main dipoles and quadrupoles. A mathematical model for LHC transient modes, such as cooldown and warmup of a magnet, a standard cell and the eight LHC sectors, has been developed on the basis of the up-to-date layout of the LHC machine, and validated by experimental data. The model considers the momentum and continuity equations, as well as the energy equations for helium and materials. Based on the simulation results, the heat transfer in the magnets has been studied and the transient modes optimized.
\end{abstract}

\section{INTRODUCTION}

The LHC cryogenic system [1] is based on a five-point feed scheme with eight refrigerators serving the eight sectors of the LHC machine [2]. Four existing refrigerators, previously working for LEP, are being upgraded from $12 \mathrm{~kW}$ to $18 \mathrm{~kW}$ at $4.5 \mathrm{~K}$ equivalent cooling power and adapted to the LHC cryogenic needs. Four new refrigerators of $18 \mathrm{~kW}$ at $4.5 \mathrm{~K}$ equivalent cooling power are being added. Within a $3.3 \mathrm{~km}$ long sector, a separate cryogenic distribution line feeds elementary cooling loops corresponding each to the length of a standard cell $(107 \mathrm{~m})$. Each refrigerator will have to cool down and warm up about 4600 tons of cold mass.

Since 1997, the previous studies on transient modes of a LHC cell [3] were carried out and the structure of LHC magnets has been updated. According to the updated structure, this paper presents a set of transient mathematical models not only suitable for a single magnet or a cell but also for a whole sector. The models have been validated by the data provided by a LHC prototype main dipole tested in February 2001. 


\section{THE UPDATED DESIGN OF LHC MAGNETS INVOLVING HEAT TRANSFER}

There are 1232 main dipoles (MB) and 400 main quadrupoles (MQ) in LHC in which helium flows through several parallel channels $(\mathrm{CH})$ of various sizes. The updated details of channels and materials used for the MB and MQ are described in TABLE 1 (where $\mathrm{D}_{\mathrm{w}}$ is the wetted perimeter, $A_{f}$ is the cross-section area, $D_{h}$ is the hydraulic diameter) and TABLE 2, whereas the location of the channels is illustrated in FIGURE 1.

TABLE 1. Channel structures of Main Dipoles and Quadrupoles

\begin{tabular}{c|ccc|ccc}
\hline \multirow{2}{*}{ Channels } & \multicolumn{3}{|c|}{ Main Dipole } & \multicolumn{3}{c}{ Main Quadrupole } \\
& $\mathrm{D}_{\mathrm{w}}[\mathrm{m}]$ & $\mathrm{A}_{\mathrm{f}}\left[\mathrm{m}^{2}\right]$ & $\mathrm{D}_{\mathrm{h}}[\mathrm{m}]$ & $\mathrm{D}_{\mathrm{w}}[\mathrm{m}]$ & $\mathrm{A}_{\mathrm{f}}\left[\mathrm{m}^{2}\right]$ & $\mathrm{D}_{\mathrm{h}}[\mathrm{m}]$ \\
\hline 1 & 0.377 & $2.83 \times 10^{-3}$ & $3.00 \times 10^{-2}$ & 2.808 & $9.30 \times 10^{-3}$ & $1.32 \times 10^{-2}$ \\
2 & 0.293 & $7.98 \times 10^{-4}$ & $1.09 \times 10^{-2}$ & 2.288 & $3.30 \times 10^{-3}$ & $5.80 \times 10^{-3}$ \\
3 & 0.248 & $5.65 \times 10^{-4}$ & $9.10 \times 10^{-3}$ & 0.372 & $5.73 \times 10^{-4}$ & $6.20 \times 10^{-3}$ \\
4 & 0.274 & $5.33 \times 10^{-4}$ & $7.80 \times 10^{-3}$ & 0.685 & $5.14 \times 10^{-4}$ & $3.00 \times 10^{-3}$ \\
5 & 0.259 & $4.22 \times 10^{-4}$ & $6.50 \times 10^{-3}$ & 0.371 & $1.85 \times 10^{-4}$ & $2.00 \times 10^{-3}$ \\
6 & 0.168 & $2.68 \times 10^{-4}$ & $6.40 \times 10^{-3}$ & 0.096 & $4.00 \times 10^{-5}$ & $1.70 \times 10^{-3}$ \\
7 & 0.355 & $3.74 \times 10^{-4}$ & $4.20 \times 10^{-3}$ & ------- & ------- & ------- \\
8 & 0.372 & $5.73 \times 10^{-4}$ & $6.20 \times 10^{-3}$ & ------ & ------ & ----- \\
9 & 0.694 & $5.16 \times 10^{-4}$ & $3.00 \times 10^{-3}$ & ------- & ------- & ----- \\
10 & 0.382 & $3.07 \times 10^{-4}$ & $3.20 \times 10^{-3}$ & ------- & -------- & ------ \\
11 & 0.341 & $3.04 \times 10^{-5}$ & $4.00 \times 10^{-4}$ & -------- & -------- & ------- \\
\hline
\end{tabular}

TABLE 2. Mass of Materials of a Main Dipole and Quadrupole

\begin{tabular}{c|cccccc}
\hline \multirow{2}{*}{ Magnets } & \multicolumn{5}{|c}{ Mass of Materials $[\mathrm{kg} / \mathrm{m}]$} \\
& Iron & Copper & Nb-Ti & Teflon & Glass & St. Steel \\
\hline Dipole & $1.18 \times 10^{3}$ & $8.11 \times 10^{1}$ & $1.95 \times 10^{1}$ & $1.78 \times 10^{1}$ & 6.87 & $5.90 \times 10^{2}$ \\
Quadrupole & $7.79 \times 10^{2}$ & $6.27 \times 10^{1}$ & $2.52 \times 10^{1}$ & $5.06 \times 10^{1}$ & 9.18 & $4.40 \times 10^{2}$ \\
\hline
\end{tabular}

Changes in materials and structure of magnets, such as replacing the previous aluminum collar material by stainless steel, led to a change of the specific heat. The present specific heat of MB has increased by about $8 \%$ in comparison with the previous design, which is shown in FIGURE 2.

\section{FLOW SCHEME AND CONSTRAINTS FOR COOLDOWN AND WARMUP}

The mass flow and cooling capacity for the cooldown and warmup of LHC magnets are supplied by the refrigerators dedicated to each sector. The refrigerators are hydraulically connected to the corresponding sector via five cooling headers. The cooldown/warmup of the magnet cold mass is performed by forced circulation of gaseous helium at decreasing/increasing temperature supplied by the refrigerators. The general flow scheme for the cooldown and warmup as well as the different regions of a LHC sector are shown in FIGURE 3.

The regular arc corresponds to the main part of the sector and is formed of 23 standard cells. The Dispersion Suppressor left and right (respectively DSL and DSR) are composed of 2 non-standard cells each. The Long Straight Section left and right (respectively LSSL and LSSR) are composed of 1 to 5 non-standard cells each. 

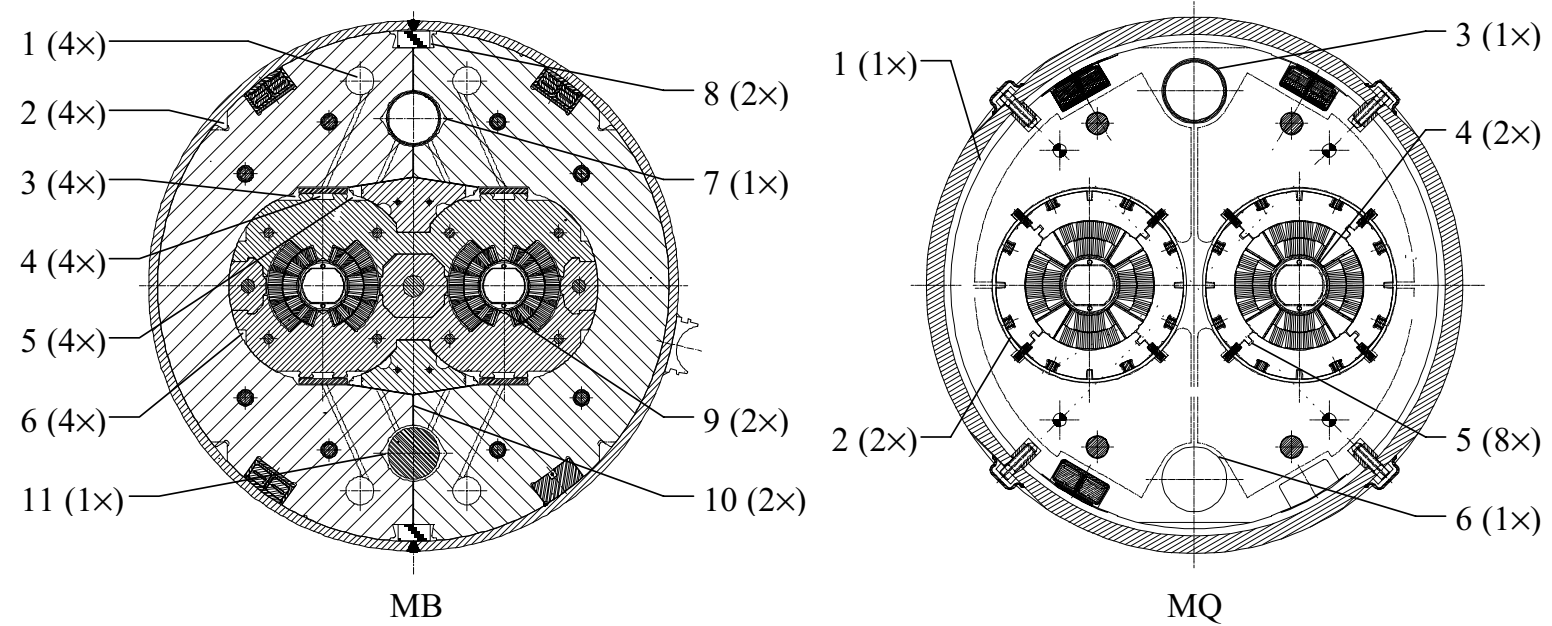

FIGURE 1. Cross-section of MB and MQ showing cooling channels

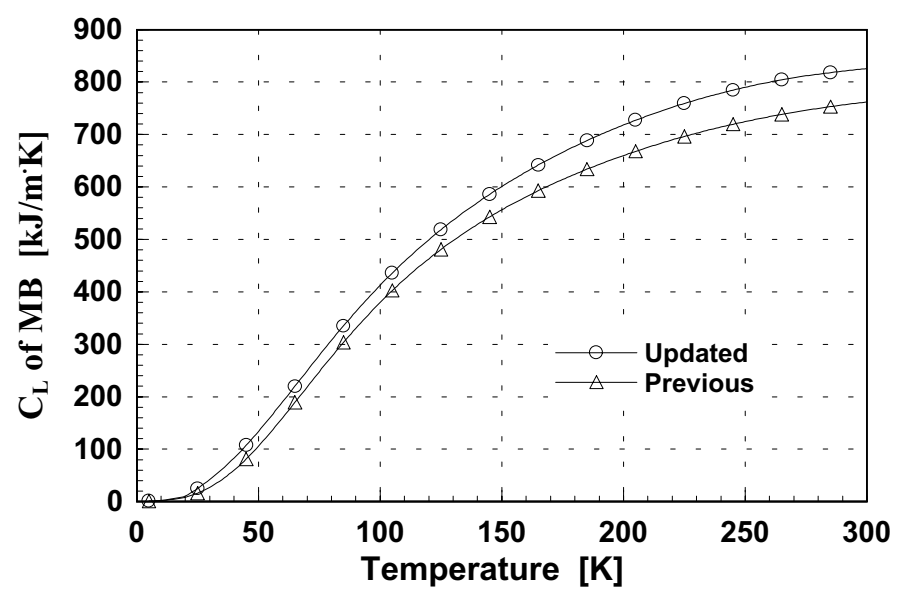

FIGURE 2. Heat capacity of material per unit length $\left(\mathrm{C}_{\mathrm{L}}\right)$ of $\mathrm{MB}$

In FIGURE 3, the helium to the magnet cold mass is supplied via header $\mathrm{C}$ and returned via header D. Headers $\mathrm{C}, \mathrm{E}$ and $\mathrm{F}$ can be interconnected using valves in order to form one single supply path. Each standard cell consists of $6 \mathrm{MBs}$ and $2 \mathrm{MQs}$. According to the cooling scheme for the standard cells, as shown in the left side of FIGURE 4, there are two types of standard cell as shown in the right side of FIGURE 4. All LHC cells are cooled down and warmed up in parallel. During a "Normal" cooldown and warmup, one refrigerator is used to supply one sector, while for a "Fast" process, two neighboring refrigerators are coupled to one sector with twice the flow and capacity.

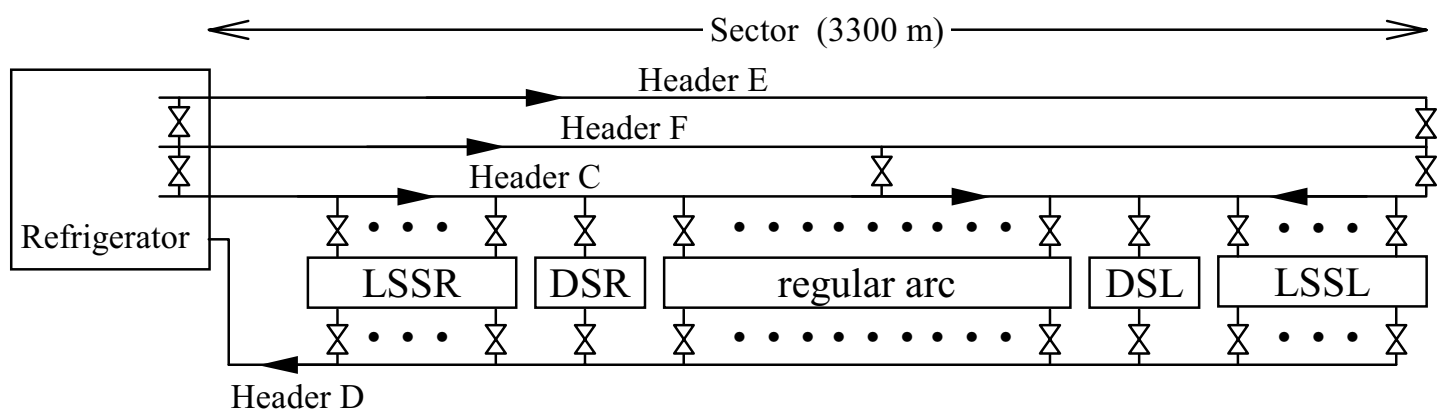

FIGURE 3. Flow-scheme for the cooldown and warmup of a LHC sector 

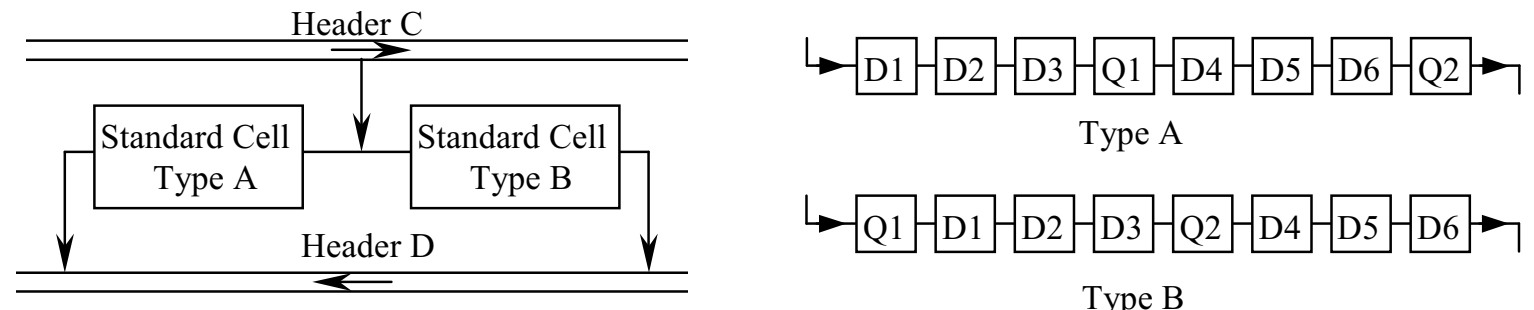

Type B

FIGURE 4. Flow scheme for two types of standard cell

The operational constrains for a sector cooldown and warmup are given in TABLE 3. To limit thermal stresses in the magnets, the boundary condition that the maximum temperature difference over any magnet must not exceed $75 \mathrm{~K}$ is introduced. To increase the cooling capacity above $80 \mathrm{~K}$, a $\mathrm{LN}_{2}$ heat exchanger of $600 \mathrm{~kW}$ is used.

TABLE 3. Constraints for cooldown and warmup of a LHC sector

\begin{tabular}{llll}
\hline & \multicolumn{2}{c}{ Operation } & Remarks \\
& Normal & Fast & \\
\hline Mass to be cooled [t] & 4600 & 4600 & \\
$\Delta$ T max. per magnet $[\mathrm{K}]$ & 75 & 75 & \\
No. of refrigerator [-] & 1 & 2 & \\
Supply headers [-] & $\mathrm{C}+\mathrm{E}+\mathrm{F}$ & $\mathrm{C}+\mathrm{E}+\mathrm{F}$ & $\mathrm{E} \& \mathrm{~F}$ for T $>80 \mathrm{~K}$ \\
Return headers [-] & $\mathrm{D}$ & $\mathrm{D}$ & \\
Max. He flow [g/s] & 770 & 1540 & available at cycle compressors \\
Max. cooling capacity $300-160 \mathrm{~K}[\mathrm{~kW}]$ & 600 & 1200 & $\mathrm{LN}_{2}$ vaporisation \\
Max. cooling capacity $160-85 \mathrm{~K}[\mathrm{~kW}]$ & 600 to 55 & 1200 to 110 & $\mathrm{LN}_{2}$ vaporisation and He turbines \\
Max. cooling capacity $85-4.5 \mathrm{~K}[\mathrm{~kW}]$ & 55 to 12 & 110 to 24 & $\mathrm{He}_{\text {He turbines }}$ \\
Max. warmup capacity $4.5-300 \mathrm{~K}[\mathrm{~kW}]$ & 600 & 1200 & electrical heaters \\
\hline
\end{tabular}

\section{MATHEMATICAL MODEL}

A simplified model for a single magnet has been developed by considering the following assumptions:

1) a one dimensional compressible flow of helium in each of the channels with zero longitudinal thermal conductivity,

2) a one dimensional wall made of several kinds of materials with infinite transverse thermal conductivity and without longitudinal heat conduction (due to the laminated structure of the cold mass)

According to these assumptions the control equations are one-dimensional and can be written as follows:

- the gas continuity equation per channel:

$$
\frac{\partial\left(\rho_{k} u_{k}\right)}{\partial x}+\frac{\partial \rho_{k}}{\partial t}=0
$$

where the subscript $k$ indicates the different channels, $\rho$ is the density of helium and $u$ its velocity and $x$ is the longitudinal dimension.

- the gas momentum equation per channel:

$$
-\frac{d p_{k}}{d x}=f_{k} \frac{1}{D_{h k}}\left(\frac{\rho_{k} u_{k}^{2}}{2}\right)
$$


where $f$ is the friction factor, $p$ is the pressure of helium.

- the energy equations for materials:

$$
\sum_{k} \alpha_{k} D_{w k}\left(T_{f k}-T_{m}\right)=\sum_{j}\left(m_{j} C_{m j}\right) \frac{\partial T_{m}}{\partial t}
$$

where $T$ is the temperature of helium or materials and the subscript $f$ refers to helium, $m$ to the materials, $j$ to the sort of the materials. The term $\sum\left(m C_{m}\right)$ represents the total heat capacity of the materials involved per unit length, $m$ is the mass of each material per unit length and $C_{m}$ is the mass specific heat of each material. The heat transfer coefficient $\alpha$ between helium and materials can be derived from the $\mathrm{Nu}$ number estimated as follows: when $\operatorname{Re} \leq 2000, \mathrm{Nu}=3.7^{[4]}$; when $\operatorname{Re} \geq 2200, \alpha$ should be determined by Dittus-Boelter Correlation, that is $\mathrm{Nu}=0.023 \mathrm{Re}^{0.8} \operatorname{Pr}^{\mathrm{n}}$, where $n$ is 0.4 for heating and 0.3 for cooling; when $2000<\operatorname{Re}<2200, \alpha$ is assumed to be proportional to $\operatorname{Re}$ and the values at the endpoints are continuous with the equations valid outside the interval.

- the energy equations for helium per channel:

$$
\frac{\partial\left(\rho_{k} u_{k} h_{k}\right)}{\partial x}+\alpha_{k} \frac{D_{w k}}{A_{f k}}\left(T_{f k}-T_{m}\right)+\frac{\partial\left(\rho_{k} h_{k}\right)}{\partial t}-\frac{\partial p_{k}}{\partial t}=0
$$

where $h$ is the enthalpy of helium.

The real gas state equation and property equations of materials have been used.

The mathematical model for the standard cell, the LHC sector and the headers can be derived from the model for a single magnet. Between each magnet there is an interconnection region, which is, in the model, represented by a node without any heat capacity. In this case the energy equation for helium is expressed as follows:

$$
\frac{\partial(\rho u h)}{\partial x}+\frac{\partial(\rho h)}{\partial t}-\frac{\partial p}{\partial t}=0
$$

To meet the requirement of a maximum temperature gradient in a magnet $(75 \mathrm{~K})$, in this model the inlet helium temperature is regulated using one or two linear ramps until the maximum cooling/warming capacity is reached.

\section{SIMULATION RESULTS AND DISCUSSION}

\section{Cooldown of a Main Dipole}

To validate the mathematical model, it was used to simulate a cooldown process of a $\mathrm{MB}$, which was tested in Feb. 2001. During the test, the flow rate was kept at $80 \mathrm{~g} / \mathrm{s}$ and no limit for the temperature difference across the magnet was set. Good agreement was found between measured and simulated results shown in FIGURE 5.

Before understanding the thermal process of a complete sector and of a cell, a normal cooldown of a single magnet has been investigated in detail. During the simulation, the mass flow rate of $30 \mathrm{~g} / \mathrm{s}$ is used and the temperature difference over the magnet is considered.

FIGURE 6 shows the details of the helium velocity and heat transfer capacity in this cooldown configuration. We can see from FIGURE 6 that larger channels have larger velocities and as a consequence higher mass flow. Due to the higher heat capacity, the largest channels contribute to the heat transfer mostly. The same phenomenon also occurs during the cooldown of $\mathrm{MQ}$, where the largest channel, $\mathrm{CH} 1$, plays the major role in heat transfer. 


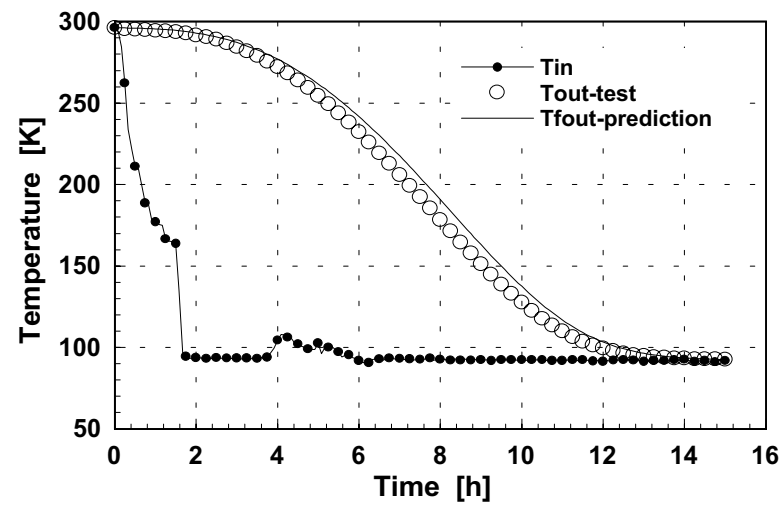

FIGURE 5. Simulation results and test data (02.2001) of MB cooldown
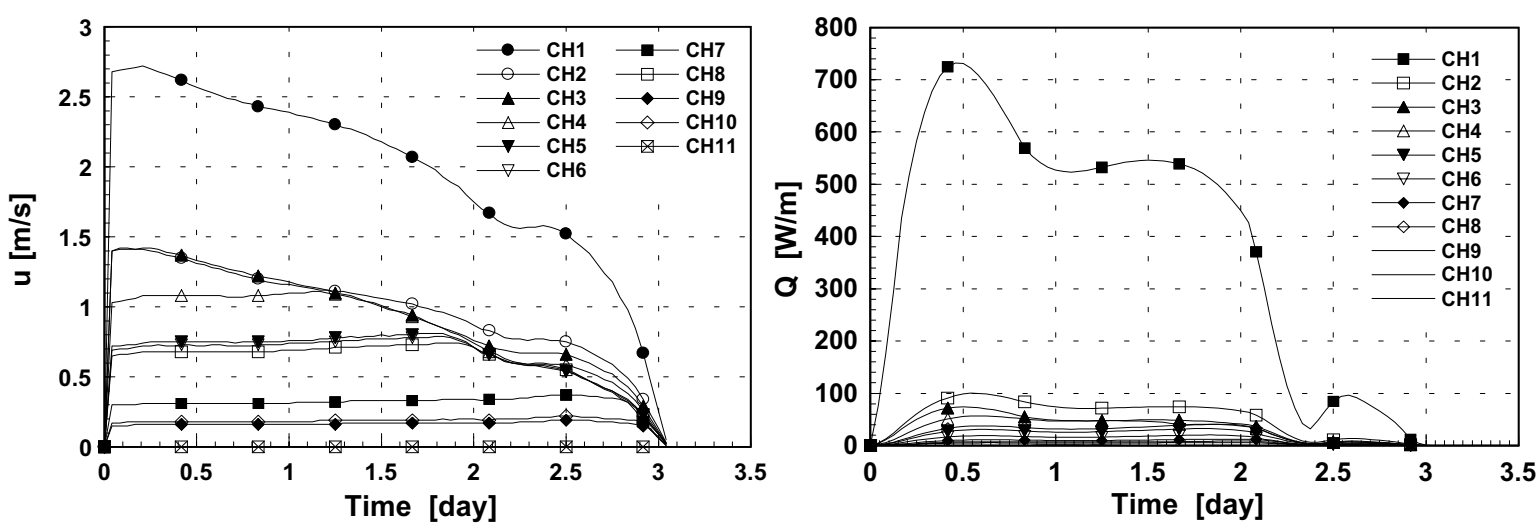

FIGURE 6. Helium velocity and heat transfer capacity at the middle of a MB during a normal cooldown

\section{Cooldown and Warmup of a Standard Cell}

FIGURE 7 and FIGURE 8 show the temperature evolutions and profiles of the cold mass for the normal cooldown of a LHC standard cell of type A and B, respectively. FIGURE 9 shows the temperature evolutions and profiles of the cold mass for the normal warmup of a LHC standard cell of type A. In these cases, the mass flow rate is $28.6 \mathrm{~g} / \mathrm{s}$, corresponding to the distribution of flow available for a sector proportional to the mass to be cooled, and the helium inlet temperature optimized to keep the maximum temperature difference along each magnet as close as possible to $75 \mathrm{~K}$ to obtain the fastest process. As a result, the cooldown process both for type A and B standard cell takes a little more than 11 days, and the warmup process less than 9.5 days.
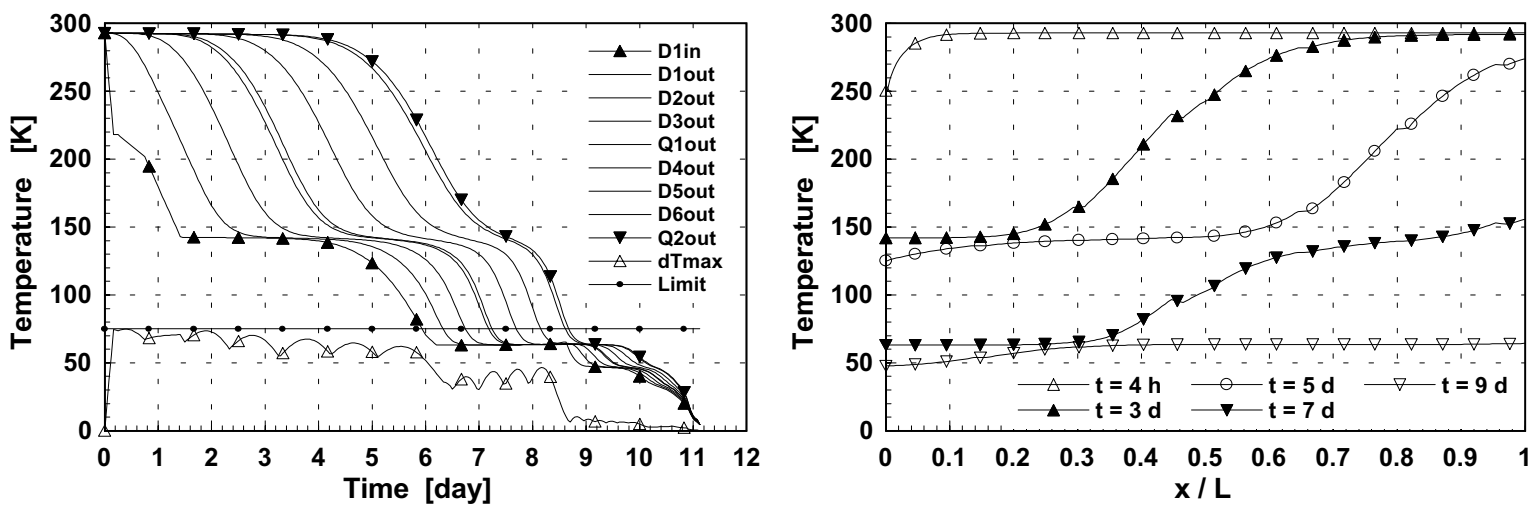

FIGURE 7. Normal cooldown of a standard cell of type A 

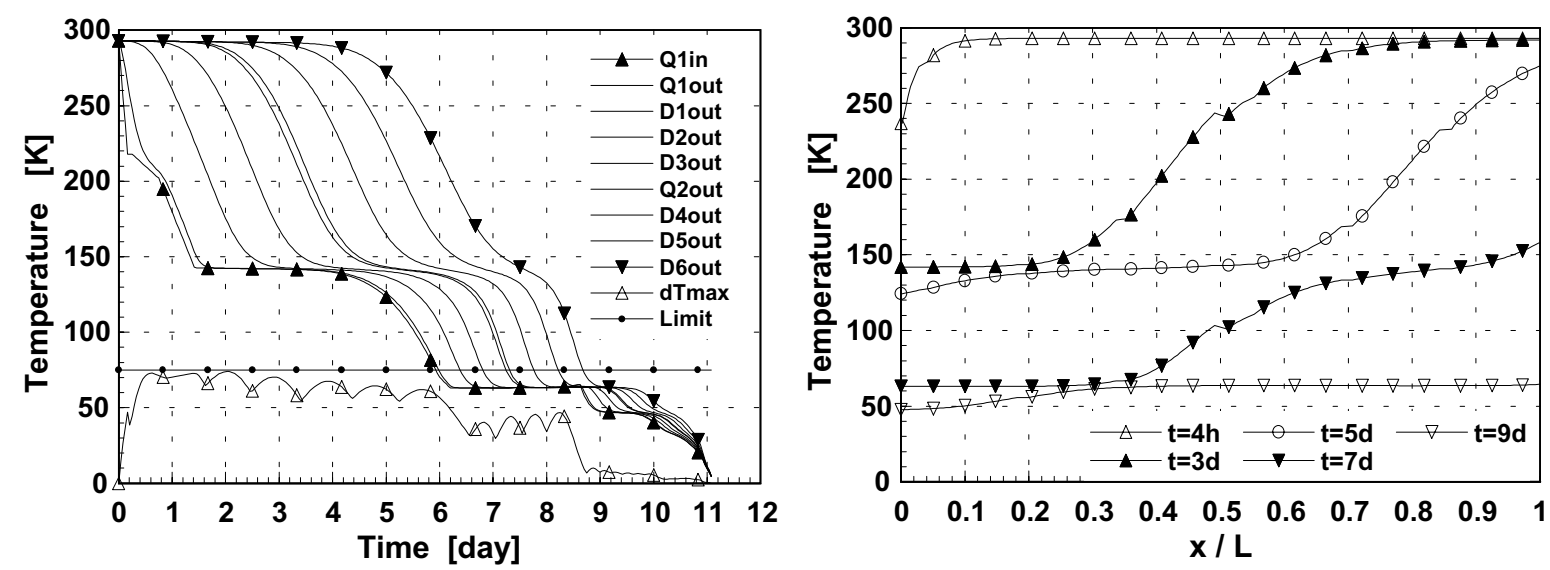

FIGURE 8. Normal cooldown of a standard cell of type B
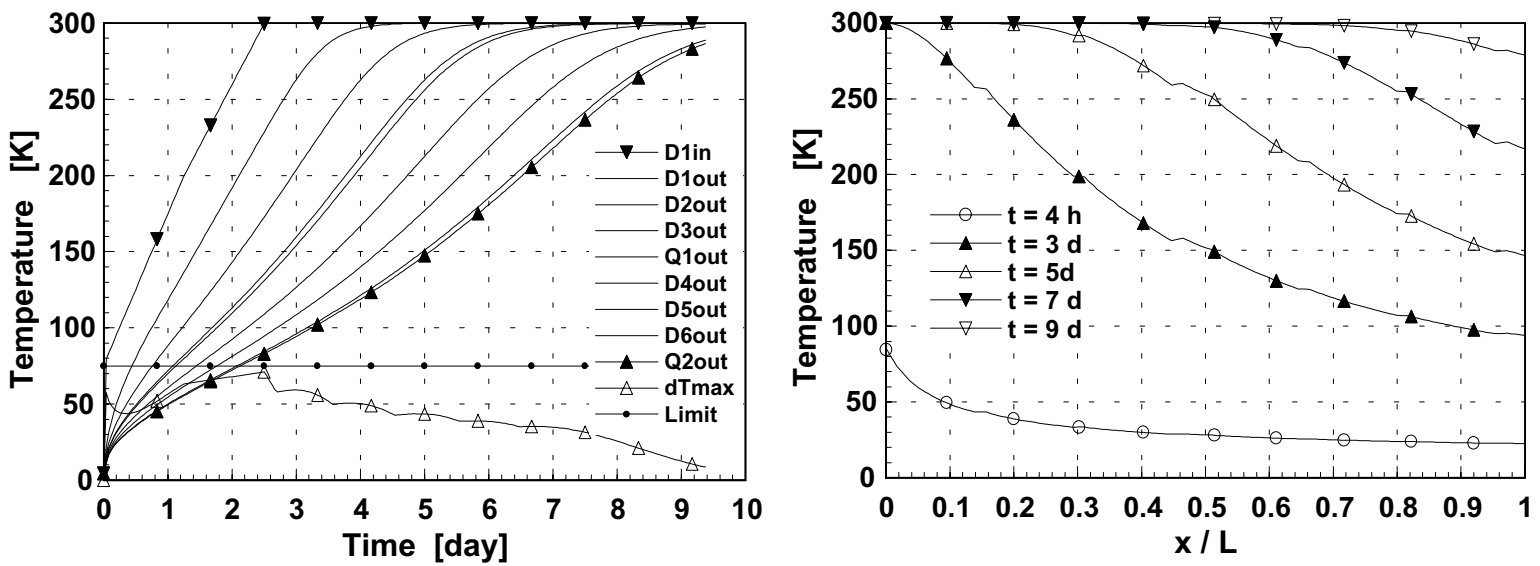

FIGURE 9. Normal warmup of a standard cell of type A

\section{Cooldown and Warmup of a Sector}

While operating a sector, attention should be paid to the flow distribution to each cell. A typical LHC sector (sector 8-1) has been studied using the model developed for the standard cell.

At first we tried to distribute the mass flow proportional to the cold mass of each cell, which resulted in a long overall cooldown time (nearly 20 days for normal cooldown). This was due to the cooldown speed of the magnets of the LSS limited by the small mass flow. Considering that the heat transfer coefficient between helium and the magnet is high and the temperature difference across each magnet cannot exceed $75 \mathrm{~K}$, the helium inlet temperature of the whole section, had to be increased accordingly. In addition, the helium inlet temperature in some small cells far away from the refrigerator was warmer due to the cooldown of the supply headers.

Therefore, we doubled the flow in the LSSR/L, increasing the flow in the two smallest cells. Following this optimization of the total mass flow distribution of $770 \mathrm{~g} / \mathrm{s}$, the flow in a standard cell is about $27.5 \mathrm{~g} / \mathrm{s}$, the flow in LSS is about $40 \mathrm{~g} / \mathrm{s}$ and in DS about $30 \mathrm{~g} / \mathrm{s}$. With this mass flow consideration, the normal cooldown of sector 8-1 takes about 12 days, the fast cooldown about $61 / 2$ days (FIGURE 10), the normal warmup about 11 days, and the fast warmup less than 6 days (FIGURE 11). 


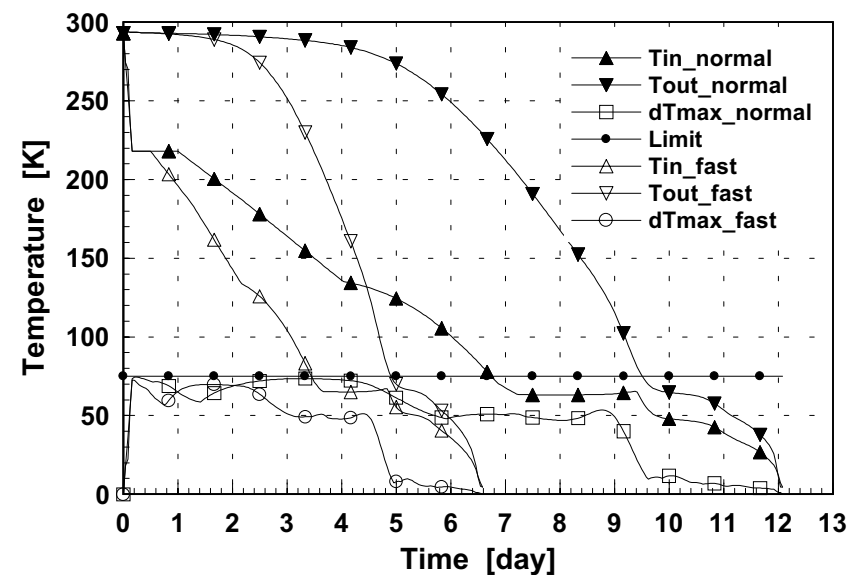

FIGURE 10. Normal and fast cooldown of sector 8-1

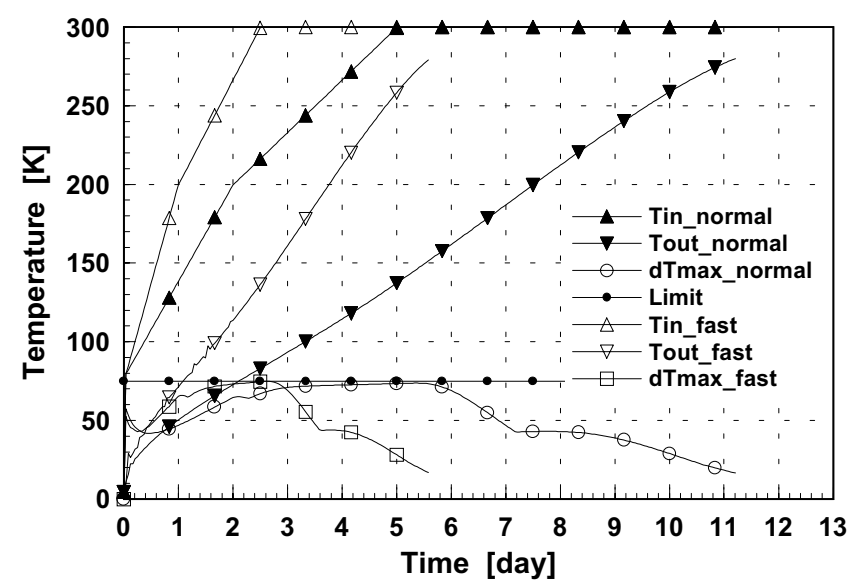

FIGURE 11. Normal and fast warmup of sector 8-1

\section{CONCLUSIONS}

The mathematical models for LHC transient modes such as cooldown and warmup of a magnet, followed by a standard cell and those for the eight LHC sectors, have been developed on the basis of the up-to-date layout of the LHC machine and validated by test data. Using the models, the simulation of cooldown and warmup of these items have been made, the heat transfer in magnets studied, and the transient modes of a typical sector have been optimized. It is possible to cool down a LHC sector in about 12 days in normal operation and in about $6 \frac{1}{2}$ days in case of fast operation. With respect to the previous estimation [3], the cooldown time of a sector has been increased by about $10 \%$.

\section{REFERENCES}

1. Lebrun, P., "Superconductivity and Cryogenics for the Large Hadron Collider," in Proc. of Beijing International Conference on Cryogenics, Beijing, 2000, pp. 28-34.

2. Evans, L. R., "The Large Hadron Collider: Present Status and Prospects," IEEE Trans. Appl. Superconductivity 10, 44-48 (2000).

3. Lebrun, P., Riddone G., and Tavian L., "Cooldown and Warmup Studies for the Large Hadron Collider," in Proc. ICEC17, Institute of Physics Publishing, London, 1998, pp. 813-816.

4. Holman, J. P., Heat Transfer $7^{\text {th }}$ ed., McGraw-Hill Book Company, London, 1992, pp. 151-159. 